https://helda.helsinki.fi

\title{
A sharp exceptional set estimate for visibility
}

\section{Orponen, Tuomas}

2018-02

Orponen , T 2018 , ' A sharp exceptional set estimate for visibility ' , Bulletin of the London Mathematical Society, vol. 50 , no. 1, pp. 1-6 . https://doi.org/10.1112/blms.12103

http://hdl.handle.net/10138/232900

https://doi.org/10.1112/blms.12103

other

acceptedVersion

Downloaded from Helda, University of Helsinki institutional repository.

This is an electronic reprint of the original article.

This reprint may differ from the original in pagination and typographic detail.

Please cite the original version. 


\title{
A SHARP EXCEPTIONAL SET ESTIMATE FOR VISIBILITY
}

\author{
TUOMAS ORPONEN
}

\begin{abstract}
A Borel set $B \subset \mathbb{R}^{n}$ is visible from $x \in \mathbb{R}^{n}$, if the radial projection of $B$ with base point $x$ has positive $\mathcal{H}^{n-1}$ measure. I prove that if $\operatorname{dim} B>n-1$, then $B$ is visible from every point $x \in \mathbb{R}^{n} \backslash E$, where $E$ is an exceptional set with dimension $\operatorname{dim} E \leq 2(n-1)-\operatorname{dim} B$. This is the sharp bound for all $n \geq 2$.

Many parts of the proof were already contained in a recent previous paper by P. Mattila and the author, where a weaker bound for $\operatorname{dim} E$ was derived as a corollary from a certain slicing theorem. Here, no improvement to the slicing result is obtained; in brief, the main observation of the present paper is that the proof method gives the optimal result, when applied directly to the visibility problem.
\end{abstract}

\section{INTRODUCTION}

For $x \in \mathbb{R}^{n}$, let $\pi_{x}: \mathbb{R}^{n} \backslash\{x\} \rightarrow S^{n-1}$ be the radial projection

$$
\pi_{x}(y):=\frac{y-x}{|y-x|} .
$$

The following theorem is the main result of the paper:

Theorem 1.1. Assume that $B \subset \mathbb{R}^{n}$ is a Borel set with $\operatorname{dim} B>n-1$. Then, there exists a set $E \subset \mathbb{R}^{n}$ with $\operatorname{dim} E \leq 2(n-1)-\operatorname{dim} B$ such that

$$
\mathcal{H}^{n-1}\left(\pi_{x}(B)\right)>0, \quad x \in \mathbb{R}^{n} \backslash E .
$$

This is the sharp bound for every $n \geq 2$.

This settles a conjecture by P. Mattila and the author in [8], where it was proven that $\operatorname{dim} E \leq n-1$ as soon as $\operatorname{dim} B>n-1$. The same conjecture had earlier appeared in Mattila's survey paper [6], see (6.1) on p. 361 Finally, the proof in the present paper also fills a small gap in the argument in [8], see the footnote on page 3 .

Remark 1.2. The strict inequality $\operatorname{dim} B>n-1$ is necessary. In fact, if $B \subset \mathbb{R}^{n}$ is purely $(n-1)$-unrectifiable with $0<\mathcal{H}^{n-1}(B)<\infty$, then it follows easily from the BesicovitchFederer projection theorem that $\mathcal{H}^{n-1}\left(\pi_{x}(B)\right)=0$ for almost every $x \in \mathbb{R}^{n}$. A more precise result is due to Marstrand [4], Theorem VI: if $B \subset \mathbb{R}^{2}$ is purely 1-unrectifiable with $0<\mathcal{H}^{1}(B)<\infty$, then $\mathcal{H}^{1}\left(\pi_{x}(B)\right)=0$ for all $x \in \mathbb{R}^{2} \backslash E$, where $\operatorname{dim} E \leq 1$. It

2010 Mathematics Subject Classification. Primary 28A75.

Key words and phrases. Hausdorff dimension, radial projections, visibility, exceptional sets.

TO is supported by the Academy of Finland through the grant Restricted families of projections and connections to Kakeya type problems.

${ }^{1}$ In fact, (6.1) in [6] states the planar version of Theorem 1.1 not as a conjecture, but a fact, which should follow from Peres and Schlag's work [10. However, Theorem 7.3 in $[10$ gives the bound $3-\operatorname{dim} B$ instead of $2-\operatorname{dim} B$ in the plane. 
is entertaining to note that when $\operatorname{dim} B>1$, the same is true with " $\mathcal{H}^{1}\left(\pi_{x}(B)\right)=0$ " replaced by " $\mathcal{H}^{1}\left(\pi_{x}(B)\right)>0 "$.

Marstrand's result can be further improved for self-similar sets: Simon and Solomyak [11] have shown that if $B \subset \mathbb{R}^{2}$ is a purely 1-unrectifiable self-similar set in the plane with $0<\mathcal{H}^{1}(B)<\infty$, and satisfying the open set condition, then $\mathcal{H}^{1}\left(\pi_{x}(B)\right)=0$ for every base point $x \in \mathbb{R}^{2}$. There is also a recent, more quantitative, version of this result by Bond, Łaba and Zahl [3].

Notation 1.3. The Grassmannian manifold of all $(n-1)$-dimensional subspaces of $\mathbb{R}^{n}$ is denoted by $G(n, n-1)$, and the Haar probability measure on $G(n, n-1)$ is denoted by $\gamma_{n, n-1}$. Given a plane $V \in G(n, n-1)$, the mapping $\pi_{V}: \mathbb{R}^{n} \rightarrow V$ is the orthogonal projection onto $V$. If $\mu$ is a Radon measure on $\mathbb{R}^{n}$, its push-forward under $\pi_{V}$ is denoted by $\pi_{V \sharp} \mu$, so that

$$
\pi_{V \sharp} \mu(B)=\mu\left(\pi_{V}^{-1}(B)\right), \quad B \subset V .
$$

For $a, b>0$, we write $a \lesssim b$, if there exists a constant $C \geq 1$ such that $a \leq C b$; the constant $C$ may, without special mention, depend on various "fixed" parameters in the proof, such as the dimension of the ambient space, or that of $B$ in Theorem 1.1 .

For $0 \leq s \leq n$, the $s$-dimensional Hausdorff measure is denoted by $\mathcal{H}^{s}$. The notation dim stands for Hausdorff dimension. Finally, if $\mu$ is a Radon measure on $\mathbb{R}^{n}$ and $0 \leq s \leq n$, the $s$-energy of $\mu$ is denoted by $I_{s}(\mu)$, so that by definition

$$
I_{s}(\mu)=\iint \frac{d \mu x d \mu y}{|x-y|^{s}} .
$$

It is well-known that, see Theorem 3.10 in [7], that

$$
I_{s}(\mu)=c(n, s) \int|\hat{\mu}(\xi)|^{2}|\xi|^{s-n} d \xi, \quad 0<s<n .
$$

\section{ACKNOWLEDGEMENTS}

This paper would not exist without the previous joint work of Pertti Mattila and myself in [8], and I am grateful for his collaboration and insights. In addition, I thank Pertti for reading the current manuscript, and giving plenty of good comments.

\section{Proof of the MAIN THEOREM}

The first part of this section contains the proof of the bound $\operatorname{dim} E \leq 2(n-1)-\operatorname{dim} B$. The second, far shorter, part discusses the question of sharpness.

3.1. Proof of the upper bound for $\operatorname{dim} E$. It suffices to prove the theorem for compact sets $B$, because if $\operatorname{dim}\left\{x: \mathcal{H}^{n-1}\left(\pi_{x}(B)\right)=0\right\}>2(n-1)-\operatorname{dim} B$ for some Borel set $B$, then also $\operatorname{dim}\left\{x: \mathcal{H}^{n-1}\left(\pi_{x}(K)\right)=0\right\}>2(n-1)-\operatorname{dim} K$ for some compact set $K \subset B$ with $n-1<\operatorname{dim} K \leq \operatorname{dim} B$. So assume that $B$ is compact. Then, the set $E:=\left\{x \in \mathbb{R}^{n}: \mathcal{H}^{n-1}\left(\pi_{x}(B)\right)=0\right\}$ is Borel, and we make the counter assumption that

$$
2(n-1)-s<\operatorname{dim} E<n-1
$$

for some $n-1<s<\operatorname{dim} B$ (such an $s$ can be found if $\operatorname{dim} E>2(n-1)-\operatorname{dim} B$ ). We may further assume that $E$ and $B$ are disjoint; if this is not true to begin with, choose two 
disjoint closed balls $B_{1}$ and $B_{2}$ such that $\operatorname{dim}\left[B \cap B_{1}\right]>s$, and $2(n-1)-\operatorname{dim}\left[B \cap B_{1}\right]<$ $\operatorname{dim}\left[E \cap B_{2}\right]$. Finally, fix $t$ strictly between $2(n-1)-s$ and $\operatorname{dim} E$, and find compactly supported Borel probability measures $\mu$ and $\nu$ inside $B$ and $E$, respectively, such that $I_{s}(\mu)<\infty$, and $I_{t}(\nu)<\infty$. Then $\mathcal{H}^{n-1}\left(\pi_{x}(\operatorname{spt} \mu)\right)=0$ for every $x \in \operatorname{spt} \nu$; to simplify notation, we assume that $B=\operatorname{spt} \mu$ and $E=\operatorname{spt} \nu$.

We briefly discuss the meaning of the assumption $\mathcal{H}^{n-1}\left(\pi_{x}(B)\right)=0$ for $x \in E$. If $L_{V, x}$ is the line perpendicular to $V \in G(n, n-1)$ and passing through $x \in \mathbb{R}^{n}$, another way to write $\mathcal{H}^{n-1}\left(\pi_{x}(B)\right)=0, x \in E$, is the following:

$$
\gamma_{n, n-1}\left(\left\{V: L_{V, x} \cap B \neq \emptyset\right\}\right)=0, \quad x \in E .
$$

This is where we needed to know that $B$ and $E$ are disjoint. Using Fubini's theorem, (3.1) implies that

$$
\nu\left(\left\{x: L_{V, x} \cap B \neq \emptyset\right\}\right)=0
$$

for $\gamma_{n, n-1}$ almost every $V \in G(n, n-1)$.

For $\delta \in(0,1)$, let $\psi_{\delta}: \mathbb{R}^{n} \rightarrow[0, \infty)$ be a radial compactly supported approximate identity (thus $\psi_{\delta}=\delta^{-n} \psi(x / \delta)$, where $\psi$ is non-negative, radial, supported on $B(0,1)$ and has integral one). Let $\mu_{\delta}:=\mu * \psi_{\delta}$, and consider the function

$$
V \mapsto f_{\delta}(V):=\int_{V} \pi_{V \sharp} \mu_{\delta} d \pi_{V \sharp} \nu, \quad V \in G(n, n-1) .
$$

We will need to know that

(i) $\left\|f_{\delta}\right\|_{L^{1}(G(n, n-1))} \geq c$ for some constant $c>0$ independent of $\delta \in(0,1)$, and

(ii) there exists $p>1$ (depending on $n, s$ and $t$ only) such that $\left\|f_{\delta}\right\|_{L^{p}(G(n, n-1))} \leq C$, where $C<\infty$ is independent of $\delta \in(0,1) 2$

In fact, (i) is precisely (3.4) in [8], so we skip the details: in brief, applying the Parseval formula and integrating in polar coordinates, one can show that $\left\|f_{\delta}\right\|_{L^{1}}$ equals a constant times $\iint|x-y|^{-(n-1)} d \mu_{\delta} x d \nu x$, which is uniformly bounded from below for $\delta \in(0,1)$.

We then prove (ii). Write $s^{\prime}:=2(n-1)-t<s$, and let $\sigma$ be a measure on $G(n, n-1)$ satisfying the growth condition $\sigma(B(x, r)) \lesssim r^{h}$ for some

$$
\max \left\{t, n-1+s^{\prime}-s\right\}<h<n-1 \text {. }
$$

Write $\mu_{V}^{\delta}:=\pi_{V \sharp} \mu_{\delta}$, and $\nu_{V}:=\pi_{V \sharp}$. Under the previous restrictions, it is known (see discussion below) that

$$
\iint_{V}|x|^{t-(n-1)}\left|\widehat{\nu_{V}}(x)\right|^{2} d \mathcal{H}^{n-1}(x) d \sigma V=c(n, t) \int I_{t}\left(\nu_{V}\right) d \sigma V \lesssim I_{t}(\nu)<\infty
$$

and

$$
\iint_{V}|x|^{s^{\prime}-(n-1)}\left|\widehat{\mu_{V}^{\delta}}(x)\right|^{2} d \mathcal{H}^{n-1}(x) d \sigma V \lesssim 1+I_{s}\left(\mu_{\delta}\right) \lesssim 1+I_{s}(\mu)<\infty .
$$

The bound (3.3) in the plane is due to Kaufman [2], and the higher dimensional analogue we need can be found in a paper by Mattila, see Lemma 5.1 in [5]. As such, the bound (3.4) is most likely due to Peres and Schlag [10, but it is certainly inspired by earlier work of Falconer [1]; a proof can also be found on p. 81 in the book [7].

\footnotetext{
${ }^{2}$ This $L^{p}$-estimate was missing from the paper 8 .
} 
Armed with Parseval, (3.3), (3.4) and Cauchy-Schwarz, we make the following estimate:

$$
\begin{aligned}
\int & \int_{V} \mu_{V}^{\delta} d \nu_{V} d \sigma V \leq \iint_{V}\left|\widehat{\mu_{V}^{\delta}}(x)\right|\left|\widehat{\nu_{V}}(x)\right| d \mathcal{H}^{n-1}(x) d \sigma V \\
= & \iint_{V}|x|^{\left(s^{\prime}-(n-1)\right) / 2}|x|^{(t-(n-1)) / 2}\left|\widehat{\mu_{V}^{\delta}}(x)\right|\left|\widehat{\nu_{V}}(x)\right| d \mathcal{H}^{n-1}(x) d \sigma V
\end{aligned}
$$

$$
\begin{aligned}
\leq & \int\left(\int_{V}|x|^{t-(n-1)}\left|\widehat{\nu_{V}}(x)\right|^{2} d \mathcal{H}^{n-1}(x)\right)^{1 / 2}\left(\int_{V}|x|^{s^{\prime}-(n-1)}\left|\widehat{\mu_{V}^{\delta}}(x)\right|^{2} d \mathcal{H}^{n-1}(x)\right)^{1 / 2} d \sigma V \\
\leq & \left(\iint_{V}|x|^{t-(n-1)}\left|\widehat{\nu_{V}}(x)\right|^{2} d \mathcal{H}^{n-1}(x) d \sigma V\right)^{1 / 2} \\
& \times\left(\iint_{V}|x|^{s^{\prime}-(n-1)}\left|\widehat{\mu_{V}^{\delta}}(x)\right|^{2} d \mathcal{H}^{n-1}(x) d \sigma V\right)^{1 / 2} \lesssim I_{t}(\nu)^{1 / 2}\left(1+I_{s}(\mu)\right)^{1 / 2} .
\end{aligned}
$$

Next, to get the $L^{p}$-result we desired, we observe that functions $g \in L^{q}(G(n, n-1))$ with $\|g\|_{L^{q}}=1$ (where $q$ is dual to $p$ ) satisfy the kind of "power bound" as was required of $\sigma$. Namely,

$$
\int_{B(V, r)} g d \gamma_{n, n-1} \leq \gamma_{n, n-1}(B(V, r))^{1 / p}\left(\int|g|^{q} d \gamma_{n, n-1}\right)^{1 / q} \lesssim r^{(n-1) / p} .
$$

So, if $p>1$ is so close to one that that $(n-1) / p \geq h$, the estimate (3.5) yields

$$
\begin{aligned}
\int f_{\delta} \cdot g d \gamma_{n, n-1} & \leq \int\left(\int_{V}\left|\widehat{\mu_{V}^{\delta}}(x)\right|\left|\widehat{\nu_{V}}(x)\right| d \mathcal{H}^{n-1}(x)\right) g(V) d \gamma_{n, n-1}(V) \\
& \lesssim I_{t}(\nu)^{1 / 2}\left(1+I_{s}(\mu)\right)^{1 / 2} .
\end{aligned}
$$

By the usual $L^{p}-L^{q}$ duality, this proves (ii).

We record two further standard facts: for $\gamma_{n, n-1}$ almost every $V \in G(n, n-1)$,

(iii) the measure $\pi_{V \sharp} \mu$ lies in the fractional Sobolev space $H^{(s-(n-1)) / 2}(V)$, and

(iv) the measure $\pi_{V \sharp} \nu$ has finite $t$-energy.

Fact (iv) follows immediately from (3.3) with $\sigma=\gamma_{n, n-1}$. Fact (iii) does not quite follow from (3.4) as stated above (because we assumed $s^{\prime}<s$ ), but it does follow from the variant of (3.4), where $s^{\prime}=s$ and $\sigma=\gamma_{n, n-1}$; this remains true, as can be proven easily via "integration in polar coordinates", see for instance (24.2) in [7]. This gives fact (iii).

Assume that $V \in G(n, n-1)$ is a plane such that (iii) and (iv) hold. Then, as observed already in [8] (or see Theorem 17.3 in the book [7]), the Hardy-Littlewood maximal function of $\pi_{V \sharp} \mu$ belongs to $L^{1}\left(\pi_{V \sharp} \nu\right)$, which implies that the functions $\pi_{V \sharp} \mu_{\delta}$ converge to a limit in $L^{1}\left(\pi_{V \sharp} \nu\right)$ both in $L^{1}\left(\pi_{V \sharp} \nu\right)$, and $\pi_{V \sharp} \nu$ almost everywhere (to see this, observe that $\pi_{V \sharp} \mu_{\delta}=\psi_{\delta}^{V} * \pi_{V \sharp} \mu$ for some approximate identity $\psi_{\delta}^{V}$ on $V$, because $\psi$ was chosen radial). We denote the limit by $g_{V} \in L^{1}\left(\pi_{V \sharp} \nu\right)$, so that

$$
\left[\pi_{V \sharp} \mu_{\delta}\right](v) \rightarrow g_{V}(v) \text { for } \pi_{V \sharp} \nu \text { almost every } v \in V \text {. }
$$

Recalling from (ii) that the sequence $\left(f_{\delta}\right)_{\delta>0}$ is bounded in $L^{p}(G(n, n-1))$ for some $p>1$, we may pick a subsequence $\left(f_{\delta_{j}}\right)_{j \in \mathbb{N}}$, which converges weakly in $L^{p}(G(n, n-1))$ 
to a limit $f \in L^{p}(G(n, n-1))$. The values of $f$ are known to us: since $\pi_{V \sharp} \mu_{\delta} \rightarrow g_{V}$ in $L^{1}\left(\pi_{V \sharp}\right)$, whenever (iii) and (iv) hold (that is, for $\gamma_{n, n-1}$ almost every $V$ ), the whole sequence $\left(f_{\delta}\right)_{\delta>0}$ converges pointwise $\gamma_{n, n-1}$ almost everywhere, and we may infer that

$$
f(V)=\int_{V} g_{V} d \pi_{\sharp} \nu \text { for } \gamma_{n, n-1} \text { almost every } V \in G(n, n-1) .
$$

On the other hand, since $f$ is the weak $L^{p}$-limit of the functions $f_{\delta_{j}}$, each of which satisfies the uniform $L^{1}$ lower bound from (i), we have

$$
\|f\|_{L^{1}(G(n, n-1))} \geq \limsup _{j \rightarrow \infty}\left\|f_{\delta_{j}}\right\|_{L^{1}(G(n, n-1))} \geq c>0 .
$$

This estimate is legitimate, because $G(n, n-1)$ is compact. It follows that $f(V)>0$ for $\gamma_{n, n-1}$ positively many planes $V \in G(n, n-1)$.

Using this fact, we find a plane $V \in G(n, n-1)$ with the following four properties: (3.2), (iii) and (iv) hold, and

$$
\int g_{V} d \pi_{V \sharp}>0
$$

The proof is finished by showing that the four conditions cannot, in fact, hold simultaneously. To this end, write

$$
G_{V}:=\left\{v \in V:\left[\pi_{V \sharp} \mu_{\delta}\right](v) \rightarrow g_{V}(v)\right\},
$$

so that $\pi_{V \sharp} \nu\left(V \backslash G_{V}\right)=0$, as we discussed after (iii) and (iv). Then, decompose the integral in (3.6) as follows:

$$
\int g_{V} d \pi_{V \sharp} \nu=\int_{\left\{v \in G_{V}: \pi_{V}^{-1}\{v\} \cap B=\emptyset\right\}} \ldots+\int_{\left\{v \in G_{V}: \pi_{V}^{-1}\{v\} \cap B \neq \emptyset\right\}} \ldots+\int_{V \backslash G_{V}} \ldots
$$

The third integral is clearly zero, and the same is true for the second integral by (3.2):

$$
\pi_{V \sharp} \nu\left(\left\{v: \pi_{V}^{-1}\{v\} \cap B \neq \emptyset\right\}\right)=\nu\left(\left\{x: L_{V, x} \cap B \neq \emptyset\right\}\right)=0
$$

But also the first integral is zero: indeed, if $v \in V$ and $\pi_{V}^{-1}\{v\} \cap B=\emptyset$, then the compactness of $B$ implies that $\pi_{V}^{-1}(B(v, \delta)) \cap B=\emptyset$ for $\delta>0$ small enough. If moreover $v \in G_{V}$, this implies that

$$
g_{V}(v)=\lim _{\delta \rightarrow 0}\left[\pi_{V \sharp} \mu_{\delta}\right](v)=0 .
$$

In other words, $g_{V}(v)=0$ for every $v \in\left\{G_{V}: \pi_{V}^{-1}\{v\} \cap B=\emptyset\right\}$. We have now seen that (3.6) cannot hold, and the ensuing contradiction completes the proof.

3.2. Sharpness of the bound. Given $n-1<s<n$, there exists a compact set $K \subset \mathbb{R}^{n}$ such that $\operatorname{dim} K=s$, and

$$
\operatorname{dim}\left\{V \in G(n, n-1): \mathcal{H}^{n-1}\left(\pi_{V}(K)\right)=0\right\}=2(n-1)-s .
$$

The example is due to Peltomäki [9], but the details can also be found in [7], Example 5.13 .

Consider the projective transformation $F: \mathbb{R}^{n} \backslash \mathbb{R}^{n-1} \rightarrow \mathbb{R}^{n}$, defined by

$$
F\left(\tilde{x}, x_{n}\right):=\frac{(\tilde{x}, 1)}{x_{n}}, \quad\left(\tilde{x}, x_{n}\right) \in \mathbb{R}^{n-1} \times \mathbb{R} \backslash\{0\} .
$$


Then $F$ maps lines in $\mathbb{R}^{n}$ of the form $\{t e+(a, 0): t \in \mathbb{R}\}$, where $a \in \mathbb{R}^{n-1}$ and $e=$ $\left(\tilde{e}, e_{n}\right) \in S^{n-1}, e_{n} \neq 0$, to lines of the form $\left\{u(a, 1)+\left(\tilde{e} / e_{n}, 0\right): u \in \mathbb{R}\right\}$. In particular, fixing the "base point" $a \in \mathbb{R}^{n-1}$, the mapping $F$ takes the lines passing through $(a, 0)$ to lines parallel to the vector $(a, 1)$. Now, let $G:=F^{-1}$, and consider the set $G(K) \subset \mathbb{R}^{n}$, which clearly still has $\operatorname{dim} G(K)=s$. The equation (3.7) can be (essentially) reworded by saying that there exists a $2(n-1)-s$ dimensional family $E$ of vectors of the form $(a, 1)$ such that $K$ lies on an $\mathcal{H}^{n-1}$-null set of lines parallel to each $(a, 1) \in E$. Hence, there exists a $2(n-1)-s$ dimensional family $E^{\prime}$ of points $a \in \mathbb{R}^{n-1}$ such that $G(K)$ lies on an $\mathcal{H}^{n-1}$-null set of lines passing through $(a, 0)$. In other words, $\pi_{(a, 0)}(G(K))=0$ for every $a \in E^{\prime}$, as desired.

\section{REFERENCES}

[1] K.J. Falconer. Hausdorff dimension and the exceptional set of projections, Mathematika 29 (1982), 109-115.

[2] R. Kaufman. On Hausdorff dimension of projections, Mathematika 15 (1968), 153-155.

[3] M. Bond, I. Łaba and J. Zahl. Quantitative visibility estimates for unrectifiable sets in the plane, appeared electronically in Trans Amer. Math. Soc. (2015)

[4] J.M. Marstrand. Some fundamental geometrical properties of plane sets of fractional dimensions, Proc. London Math. Soc. (3) 4 (1954), 257-302

[5] P. Mattila. Hausdorff dimension, orthogonal projections and intersections with planes, Ann. Acad. Sci. Fenn. A Math. 1 (1975), 227-244.

[6] P. Mattila. Hausdorff dimension, projections, and the Fourier transform, Publ. Mat. 48 (2004), $3-48$

[7] P. Mattila. Fourier Analysis and Hausdorff Dimension, Cambridge University Press, Cambridge, 2015.

[8] P. Mattila and T. Orponen. Hausdorff dimension, intersections of projections and exceptional plane sections, appeared electronically in Proc. Amer. Math. Soc. (2015), available at arXiv:1509.05724

[9] A. Peltomäki. Projektiot ja Hausdorffin dimensio, Licenciate thesis, Helsingin yliopisto (1988)

[10] Y. Peres and W. Schlag. Smoothness of projections, Bernoulli convolutions, and the dimension of exceptions, Duke Math. J. 102 (2000), 193-251.

[11] K. Simon and B. Solomyak. Visibility for self-similar sets of dimension one in the plane, Real Anal. Exchange 32(1) (2006/2007), 67-78 